\title{
Self-Calibration Phenomenon for Near-Infrared Clinical Measurements: Theory, Simulation, and Experiments
}

\author{
Idit Feder, Hamootal Duadi, Ruchira Chakraborty, and Dror Fixler*i)
}

Faculty of Engineering and the Institute of Nanotechnology and Advanced Materials, Bar Ilan University, Ramat Gan 5290002, Israel

Supporting Information

ABSTRACT: An irradiated turbid medium scatters the light in accordance to its optical properties. Near-infrared (NIR) clinical methods, which are based on spectral-dependent absorption, suffer from an inherent error due to spectral-dependent scattering. We present here a unique spatial point, that is, iso-pathlength (IPL) point, on the surface of a tissue at which the intensity of re-emitted light remains constant. This scattering-indifferent point depends solely on the medium geometry. On the basis of this natural phenomenon, we suggest a novel optical method for self-calibrated clinical measurements. We found that the IPL point exists in both cylindrical and semi-infinite tissue geometries (Supporting Information, Video file). Finally, in vivo human finger and mice measurements are used to validate the crossing point between the intensity profiles of two wavelengths. Hence, measurements at the IPL point yield an accurate absorption assessment while eliminating the scattering dependence. This finding can be useful for oxygen saturation determination, NIR spectroscopy, photoplethysmography measurements,

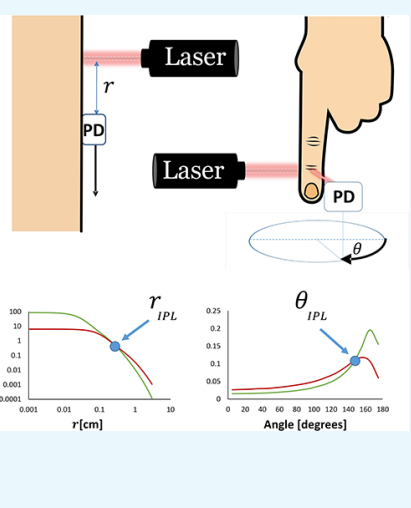
and a wide range of optical sensing methods for physiological aims.

\section{INTRODUCTION}

Diagnosis of a physiological state by extracting optical parameters of tissues, as implemented in near-infrared (NIR) spectroscopy, optical coherence tomography, fluorescence, etc., $^{1-5}$ is widespread. However, human tissue, which is a turbid medium, poses a challenge for the different optical methods. Light reflectance from a tissue has spectral-dependent absorption and scattering. ${ }^{6}$ Hence, evaluating absorptiondependent physiological parameters requires calibration of the scattering to obtain accurate information. Methods for extracting optical properties from the reflected light are based on either the amplitude in multiple sources or multiple point measurements. Most methods are complex and based on modulated sources with phase, time, and frequency analyses. ${ }^{7}$

Pulse oximetry is one of the most common point-of-care tests because it is noninvasive, characterized by high speed, and can be interpreted even without a physician. It uses two light sources for extracting blood saturation, pressure, and perfusion on the basis of ratiometric absorption measurements. The change in optical properties due to wavelength produces a difference in the optical path. Hence, at different wavelengths, a position on the surface of the medium has an intensity that is influenced by a different optical path.

The full scattering profile (FSP), which is the angular intensity distribution, ${ }^{8,9}$ is a new point of view for extracting optical properties of cylindrical tissues using a simple system containing a continuous wave source and a photodetector (PD). The FSP of cylindrical tissues is relevant to biomedical measurement of fingers, earlobes, or pinched tissues. Using the FSP, the iso-pathlength (IPL) point phenomenon is revealed; an IPL point is a point on the surface of a cylindrical medium where the light intensity remains constant and does not depend on the reduced scattering coefficient of the medium but on the spatial structure. This phenomenon serves as a self-calibration point because the intensity there is influenced by absorption regardless of the scattering and thus improves the accuracy of the optical property extraction. Moreover, we presented a linear dependency between the radius of the tissue and the point's location.

Most existing instruments do not deal with the FSP but rather focus only on the reflection or transmission of light. The novel finding of the IPL point, to the best of our knowledge, has not been reported before this research. However, we have found supporting reports on reflection from a semi-infinite medium in diffusion reflection $(D R)^{10,11}$ and reflection phase measurements. ${ }^{12}$ Mourant $^{10}$ reported on a source-detector separation that does not depend on scattering properties at the range close to the light source. Mourant presented a Monte Carlo (MC) simulation and phantom experiments of reflected light from a semi-infinite medium. Moreover, Calabro showed the influence of the phase function on generalized diffuse reflectance models ${ }^{13}$ from a semi-infinite medium and presented a singular distance from the source, below the diffusion regime, which is insensitive to the phase function. Savo et al. ${ }^{14}$ recently presented a research that supports our work. They reported that the light mean path length is invariant with respect to the microstructure of the medium. The work was based on the random walk theory, MC simulations, and measurements of scattered light from glass tubes with scattering

Received: January 3, 2018

Accepted: February 22, 2018

Published: March 8, 2018 
solutions using an autocorrelator for extracting the mean path length.

We believe that this natural phenomenon of a point, which is indifferent to changes in the scattering property, depends solely on the tissue geometry. Knowledge of the position of this point in each geometry will allow an accurate, self-calibrated detection of absorption-dependent physiological parameters. Note that this phenomenon only occurs when multiple scattering exists. ${ }^{6}$

We show this phenomenon from a semi-infinite medium theoretically, by simulations, and experimentally. Diffusion theory is supported by $\mathrm{MC}$ simulation results as well as the experimental results with phantoms. We consider two geometries, a cylindrical model and a semi-infinite model, and present a point that is not dependent on the scattering coefficient. Both geometries are validated by in vivo measurements, a human finger for the cylindrical model and a mouse neck for the semi-infinite model.

The finding of the IPL point can be useful for oxygen saturation determination, NIR spectroscopy, photoplethysmography (PPG) measurements, and a wide range of optical sensing methods for physiological aims in a more accurate way. The FSP utilizes the entire distribution profile rather than just parts of it by the complete characterization of the material and its geometry, thereby improving the existing systems and creating new methods.

\section{DIFFUSION THEORY SIMULATION}

The propagation of light through a tissue is mostly described by the radiative transport equation (RTE). RTE is an approximation to Maxwell's equations, based on the conservation of energy, describing the propagation of energy

$$
\begin{aligned}
\frac{1}{c} \frac{\partial L(r, \hat{s}, t)}{\partial t}= & -\hat{S} \nabla L(r, \hat{s}, t)-\mu_{\mathrm{t}} L(r, \hat{s}, t) \\
& +\mu_{\mathrm{s}} \int_{0}^{4 \pi} L(r, \hat{k}, t) P\left(\hat{s}^{\prime} \cdot \hat{s}\right) \mathrm{d} \Omega \\
& +S(r, \hat{s}, t)
\end{aligned}
$$

The radiation $(L(r, \hat{s}, t))$ transported by photons in direction $\hat{s}$ and to a distance $r$ from the source at time $t$ is represented by eq 1 , where $c$ is the speed of light. The extinction coefficient is $\mu_{\mathrm{t}}=\mu_{\mathrm{a}}+\mu_{\mathrm{s}}^{\prime}$, where $\mu_{\mathrm{s}}^{\prime}$ is the reduced scattering coefficient defined by $\mu_{\mathrm{s}}^{\prime}=(1-g) \mu_{\mathrm{s}}$ for anisotropy factor $g$ and scattering coefficient $\mu_{\mathrm{s}} . P\left(\hat{s}^{\prime}, \hat{s}\right)$ is the phase function, which gives the probability for a photon traveling in direction $\hat{s}$ to be scattered in direction $\hat{s}^{\prime} . \Omega$ is a solid angle, and $S(r, \hat{s}, t)$ represents the radiant source in the tissue.

The radiation integrated over all directions is defined by the fluence rate $\left[\mathrm{W} / \mathrm{cm}^{2}\right]^{15}$

$$
\phi(r, t)=\int_{0}^{2 \pi} L(r, \hat{s}, t) \mathrm{d} \hat{s}
$$

The most typical approach for solving the RTE analytically is the diffusion approximation. ${ }^{16,17}$ Using the solution of diffusion theory at steady state, we can get the expression for fluence $\psi(r)\left[\mathrm{J} / \mathrm{cm}^{2}\right]$ in response to power $Q$ located at $r=0$ as follows

$$
\psi(r)=\int_{t=0}^{\infty} \phi(r, t) \mathrm{d} t=Q \frac{\exp \left(-\frac{r}{\delta}\right)}{4 \pi \mu_{\mathrm{a}} \delta^{2} r}=Q \frac{\exp \left(-\frac{r}{\delta}\right)}{4 \pi D r}
$$

Equation 3 describes the steady-state diffusion of light from a point source of power $P$, where $r$ is the position on the $X Y$ plane, $D$ is the diffusion length, $D=1 /\left(3\left(\mu_{\mathrm{a}}+\mu_{\mathrm{s}}^{\prime}\right)\right)$, and $\delta$ is the optical penetration depth, $\delta=\sqrt{D / \mu_{\mathrm{a}}}$. We determine $Q$ to be 1 to examine the light transport independent of the source's power.

Because we are interested in developing light diffusion equations on the surface of a cylindrical tissue, we express the fluence on the edge of a circle and obtain the FSP at a cross section, with radius $a$, of the illuminated cylinder. We can define distance $r$ of a point on the circumference from the light source (Figure 1) by the law of cosines as follows

$$
r=a \sqrt{2(1+\cos \theta)}
$$

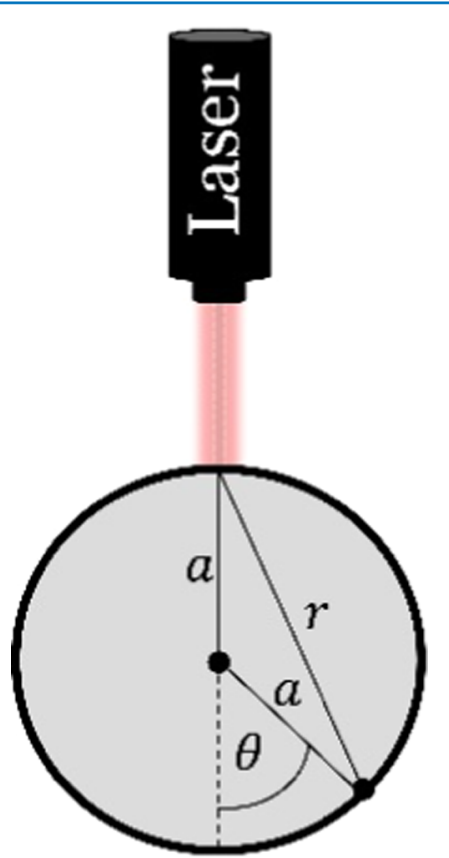

Figure 1. Geometric description of a point on the circumference in a circular cross section.

On the basis of eqs 3 and 4, the steady-state diffusion of light on the surface of the cylinder is obtained. This fluence in accordance with $\mu_{\mathrm{s}}^{\prime}$ for each $\theta$ at the circle surface is represented by the following equation

$$
\begin{aligned}
& \psi(r)=\frac{\exp \left(-\frac{r}{\delta}\right)}{4 \pi D r} \\
& =\frac{3\left(\mu_{\mathrm{a}}+\mu_{\mathrm{s}}^{\prime}\right) \exp \left(-a \sqrt{6 \mu_{\mathrm{a}}(1+\cos \theta)\left(\mu_{\mathrm{a}}+\mu_{\mathrm{s}}^{\prime}\right)}\right)}{-4 a \pi D \sqrt{2(1+\cos \theta)}}
\end{aligned}
$$

The change of the fluence per $\theta$ can predict $\theta_{\text {IPL }}$, where $\frac{\partial \psi(r)}{\partial \theta}=0$.

$$
\theta_{\mathrm{IPL}}=\arccos \left(\frac{1}{6 a^{2} \mu_{\mathrm{a}}\left(\mu_{\mathrm{a}}+\mu_{\mathrm{s}}^{\prime}\right)}-1\right)
$$

For the relevant $\mu_{\mathrm{s}}^{\prime}$ range, the fluence depends much more on the cylindrical radius $a$ than on $\mu_{\mathrm{s}}^{\prime}$ (Figure 2). Because we assume an angular accuracy of $5^{\circ}$, all of the $\mu_{\mathrm{s}}^{\prime}$ yield an identical angle. 


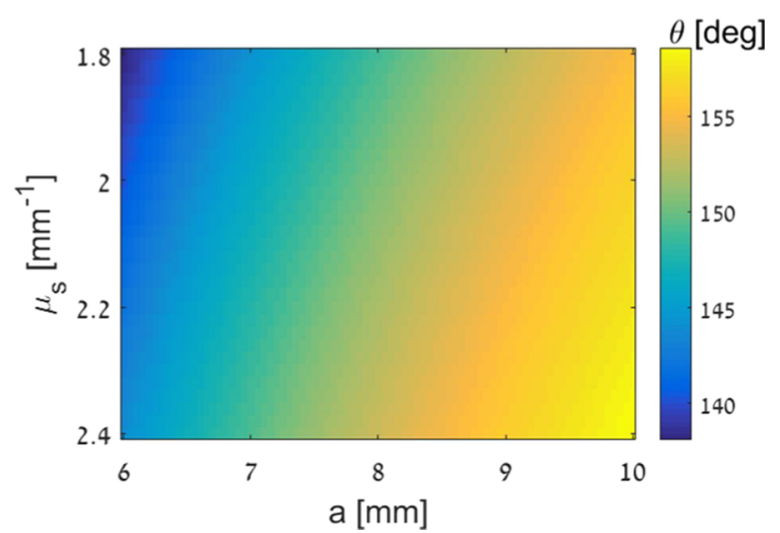

Figure 2. Dependency of $\theta_{\mathrm{IPL}}$ on radius $a$ and reduced scattering coefficient $\mu_{\mathrm{s}}^{\prime}$. $\mu_{\mathrm{s}}^{\prime}$ has a smaller influence on the $\theta_{\mathrm{IPL}}$ value (less than $5^{\circ}$ ) than the radius $a$.

\section{RESULTS}

3.1. Full Scattering Profile of a Cylindrical Medium: Theory, Simulation, and Experiment Results. The FSPs of a cylindrical medium based on diffusion theory are presented in Figure 3. The reduced scattering coefficients are in the human skin range of $15-30 \mathrm{~cm}^{-1}$ and the radius $1 \mathrm{~cm}$. The IPL point appears at $127^{\circ}$.

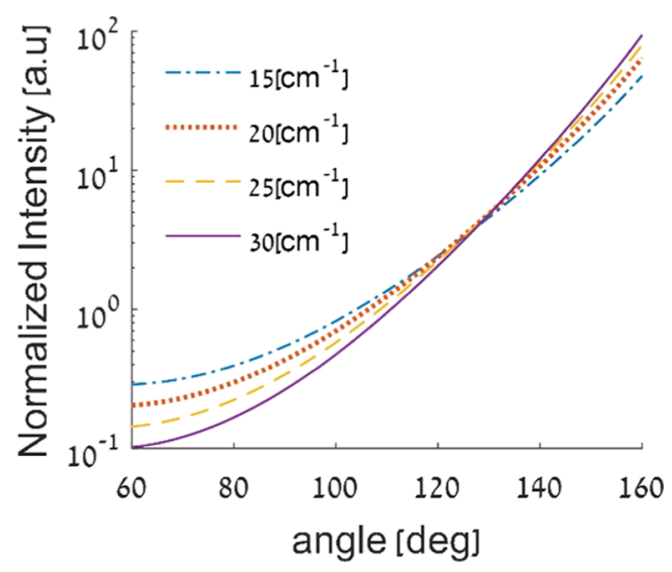

Figure 3. FSPs and the IPL point of cylindrical tissues according to diffusion theory (logarithmic scale).

The location of the IPL point on the surface of a cylinder is dependent on its radius. As the radius increases, the fixedintensity point becomes closer to the light source. Because diffusion theory is relevant for distances larger than one or two transport mean free path (MFP') from the light source, ${ }^{15}$ where $\mathrm{MFP}^{\prime}=1 /\left(\mu_{\mathrm{a}}+\mu_{\mathrm{s}}^{\prime}\right)$. The radii smaller than the MFP' are not valid for this theory, and similar is the case with the large radii where the IPL point appears at high angles because diffusion theory is inaccurate near the source. At the relevant range of radii, which describes fingers and cylindrical tissues that the light can penetrate through, the IPL point depends on the tissue radius.

Using our MC simulation, which is appropriate for the regions close to the source, we described the FSPs of cylindrical tissues with different reduced scattering coefficients of 14 to 26 $\mathrm{cm}^{-1}$ for $6.5 \mathrm{~mm}$ tissue radius, as shown in Figure 4a, and simulated these FSPs for various tissue diameters. The IPL point appeared in each set of tissues. Moreover, a linear

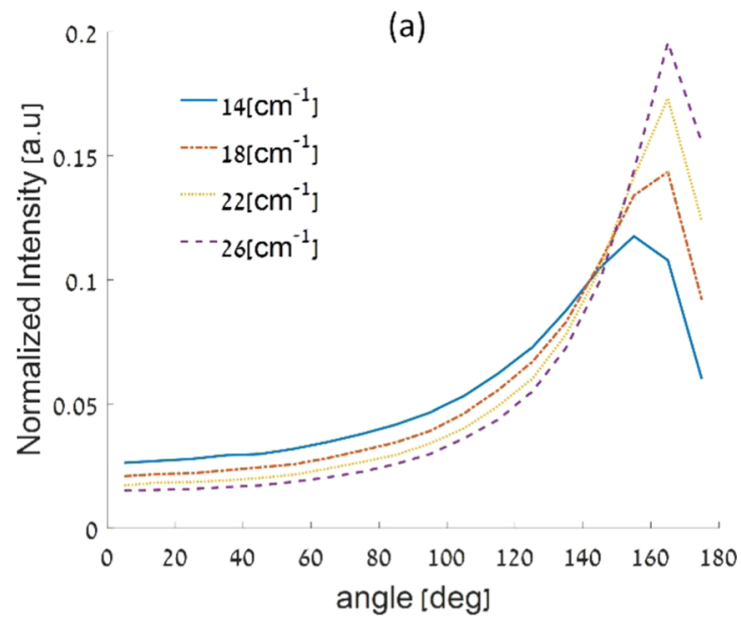

(b)

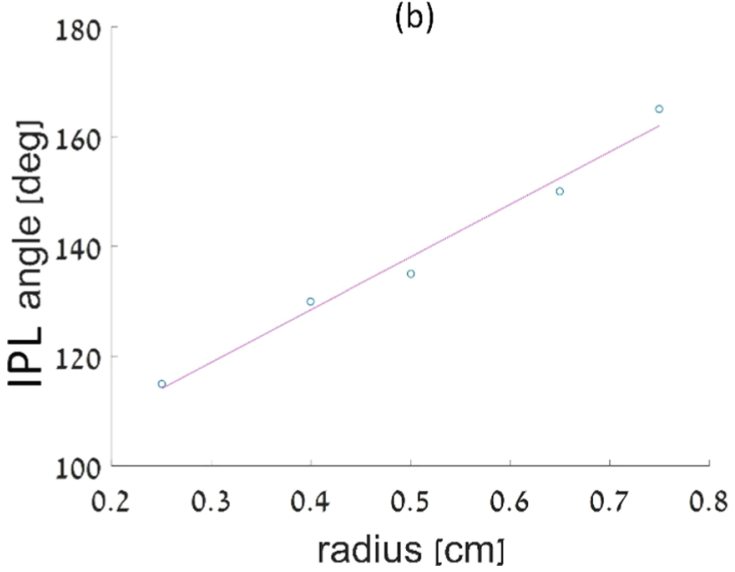

Figure 4. (a) FSPs and the IPL point from the MC simulation of a cylindrical finger tissue, $6.5 \mathrm{~mm}$ radius. (b) Linear dependency of IPL point on the radius.

dependency on tissue radius was determined, as shown in Figure $4 \mathrm{~b}$.

We built a unique setup (Figure 5) for noninvasive encircled measurements containing a continuous wave source and a portable photodetector (PD).

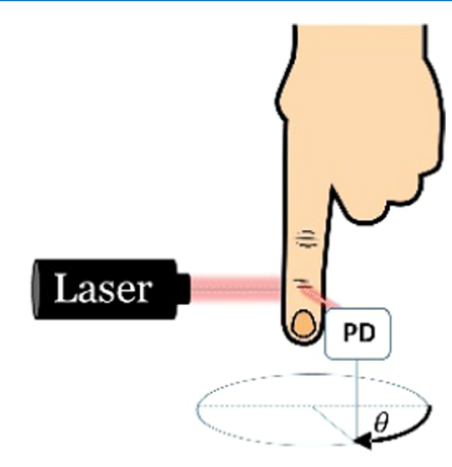

Figure 5. Illustration of the setup for measuring FSP using a portable photodetector (PD).

Our experiment reveals an IPL point, in accordance with the simulation, that is independent of the optical properties. We used one continuous wave laser, a photodetector, and tissuemimicking phantoms presenting different optical properties. The FSP and the IPL point that appeared at $115^{\circ}$ are shown in 
Figure 6a. The linear dependence of the IPL point location on the radius of the cylindrical phantom is presented in Figure $6 \mathrm{~b}$.
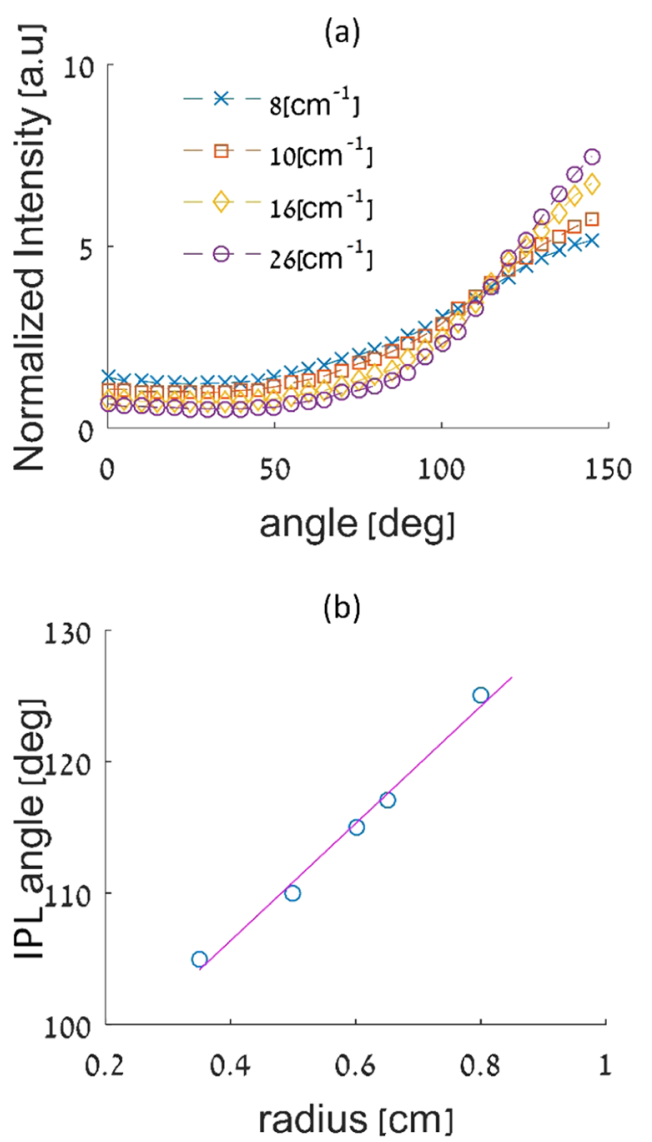

Figure 6. Experimental results of an IPL point in a cylindrical medium. (a) FSPs of phantoms, with $0.6 \mathrm{~cm}$ radius and (b) linear dependency of IPL point on the radius.

We measured a human finger in vivo in our system to validate the FSP behavior of cylindrical tissues. We used two wavelengths and obtained the FSPs of a human finger, which are presented in Figure 7 . The effective radius of the finger is $0.6 \mathrm{~cm}$, and the common point for these FSPs appears at $110^{\circ}$, whereas in the phantom results, at a similar effective radius, the IPL point is obtained at $115^{\circ}$. The human finger contains blood vessels, which add a non-negligible absorption. The absorption

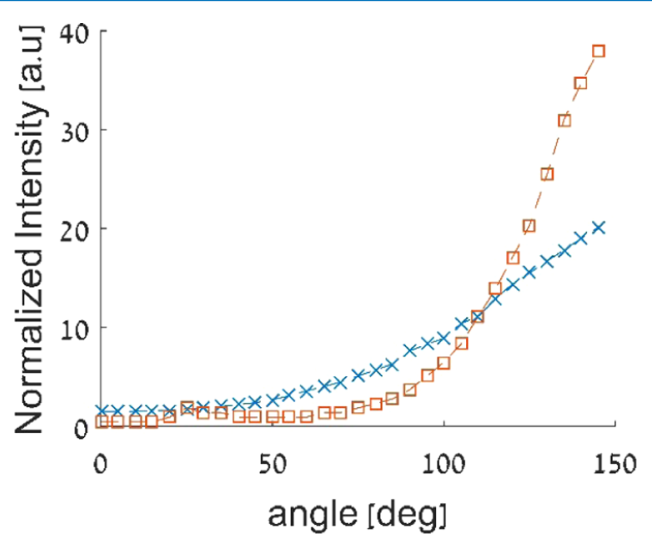

Figure 7. FSP of a male index finger in 650 (crosses) and $780 \mathrm{~nm}$ (squares). has a spectral dependence; therefore, the measurements are decreased for each wavelength separately and the common point is different from the phantoms' IPL point.

3.2. Reflection from a Semi-Infinite Medium: Theory, Simulation, and Experiment Results. On the basis of diffusion theory, we calculated the reflected light intensity from a semi-infinite medium at distance $r$ from the source. The reflection intensity from a semi-infinite medium with different reduced scattering coefficients, in the range of $15-30 \mathrm{~cm}^{-1}$, is shown in Figure $8 \mathrm{a}$. A crossing point appears at $8 \mathrm{~mm}$ from the source. This location can also be called the IPL distance, $r_{\text {IPL }}$ from the illumination point.
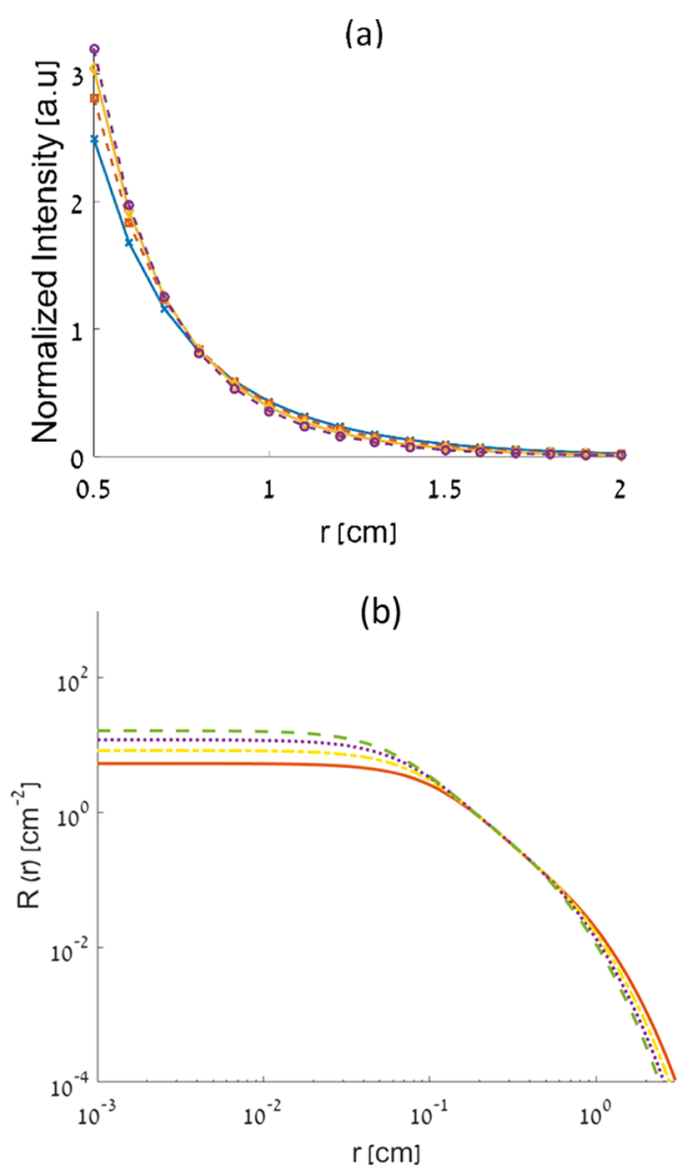

Figure 8. Reflection from a semi-infinite tissue: (a) reflection according to diffusion theory; a crossing point does not depend on scattering properties (blue crosses, orange squares, yellow diamonds, and purple circles represent $\mu_{\mathrm{s}}^{\prime}$ at $15,20,25$, and $30 \mathrm{~cm}^{-1}$, respectively). (b) MC simulation of a crossing point, which is indifferent to changes in the scattering property (orange solid, yellow dash-dotted, magenta dotted, and green dashed lines represent $\mu_{\mathrm{s}}^{\prime}$ at 8 , 10,12 , and $14 \mathrm{~cm}^{-1}$, respectively).

In addition, a MC simulation for various reduced scattering coefficients, in the range of $8-14 \mathrm{~cm}^{-1}$, suggested that path lengths do not depend on the scattering properties of the medium at a distance of $r_{\mathrm{IPL}}=3.5 \mathrm{~mm}$ from the source, as presented in Figure $8 \mathrm{~b}$. These values represent the optical tissue coefficients, as we used in the experimental system.

We conducted an experiment on DR measurements from a solid silicon-based phantom without absorption components. ${ }^{18,19}$ An illustration of the measurement system is shown in Figure 9. 


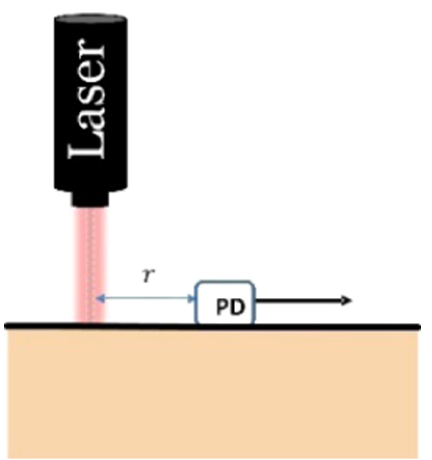

Figure 9. Illustration of a setup for DR measurement from a semiinfinite tissue with a PD.

By measuring two wavelengths, we found a common point at a specific distance from the light source, as presented in Figure 10a. The common light intensity for two different wavelengths clearly represents the area that does not depend on the scattering properties.

In addition, we measured a semi-infinite medium in vivo. The light intensity as a function of distance from the light source, which was reflected from the carotid artery of mice, is presented in Figure 10b. The common light intensity point for the mice appears closer to the light source than that in the control phantom. This is expected because the absorption through tissues is higher in the red range than that in the NIR range. ${ }^{20}$ Hence, the phantom measurements reveal the IPL point where scattering changes have a minimal impact without absorption components. By detecting this location, we can achieve accurate extraction of absorption coefficients from in vivo measurements, whereas the measurement at the right point excludes the scattering.

\section{DISCUSSION}

We claim that the finding of an IPL point in cylindrical (Figure 11a) and semi-infinite (Figure 11b) tissues is actually the same phenomenon. Both cases are geometric positions that depend on the distance from the light source where the optical path length is not affected by the optical parameters.

The relation between the angle of an IPL point in a cylindrical medium, $\theta_{\text {IPL, }}$ and the radius $a$ will be obtained merely by geometrical computations. Let us consider both geometries on the same coordinate system (Figure 11c).

In the semi-infinite geometry, the IPL distance, $r_{\text {IPL }}$, from the illumination point inside the medium defines the following circle equation (blue-dashed line in Figure 11b)

$$
x^{2}+y^{2}=r_{\text {IPL }}^{2}
$$

In the cylindrical geometry, the tissue cross section is placed adjacent to the light source (black circle in Figure 11a); hence, the surface is defined by

$$
x^{2}+(y+a)^{2}=a^{2}
$$

We calculated the intersection between eqs 7 and 8 and expressed it in terms of the IPL angle, $\theta_{\text {IPL }}$ using the Taylor approximation for arccosine

$$
\theta_{\mathrm{IPL}}=\frac{\pi}{2}+\left(1-\frac{\frac{r_{\mathrm{IPL}}^{2}}{2}}{a^{2}}\right) \frac{180}{\pi}
$$

(a)

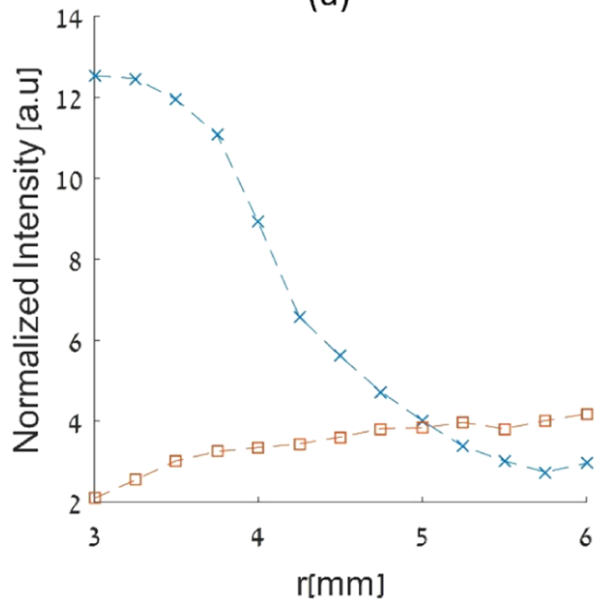

(b)

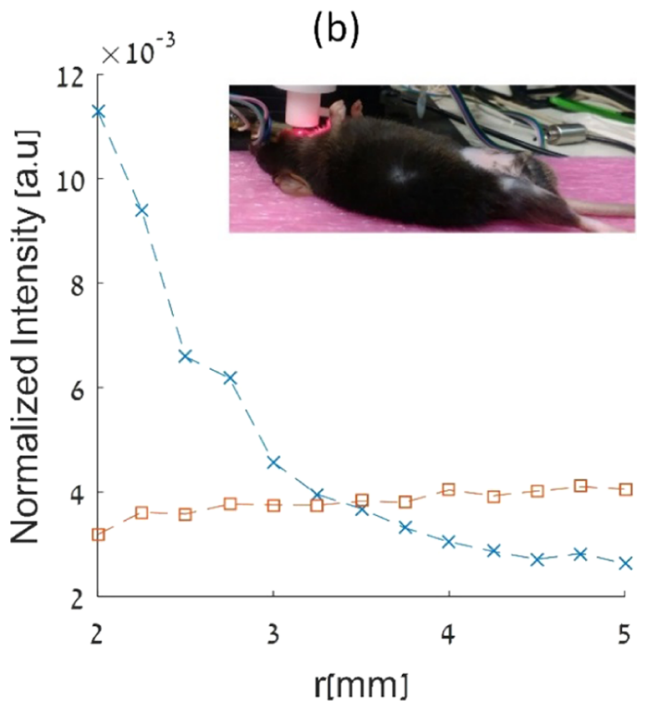

Figure 10. (a) DR measurements of phantom using two wavelengths; a crossing point appears at $5.1 \mathrm{~mm}$ (crosses for $650 \mathrm{~nm}$ and squares for $780 \mathrm{~nm}$ ). (b) In vivo DR measurements of mouse (in inset) using two wavelengths; a crossing point appears at $3.4 \mathrm{~mm}$ (crosses for 650 $\mathrm{nm}$ and squares for $780 \mathrm{~nm}$ ).

In Figure 11d, the black line represents this equation for $r_{\mathrm{IPL}}=$ $6.4 \mathrm{~mm}$ and the relevant range of $a$. This relation is compared with the experimental results of human fingers where $\theta_{\text {IPL }}$ was measured by a single wavelength by comparing two perpendicular orientations (dots in Figure 11d from ref 22). The relation from eq 9 shows a high fit to these experimental results. Hence, the intersection found in eq 9 reveals the $\theta_{\text {IPL }}$ angle in which the IPL point is located according to the finger diameter.

Note that the $r_{\text {IPL }}$ that presented high correlation to the in vivo results from ref 22 is a value similar to $r_{\text {IPL }}=6.8 \mathrm{~mm}$ from diffusion theory. Hence, we can determine that the IPL points, which are not influenced by the scattering properties of the medium in the two mentioned geometries, are converged to the same distance from the light source (Supporting Information, Video file).

\section{CONCLUSIONS}

The optical measurements of tissues are influenced by the absorption as well as scattering. Therefore, there is a challenge 

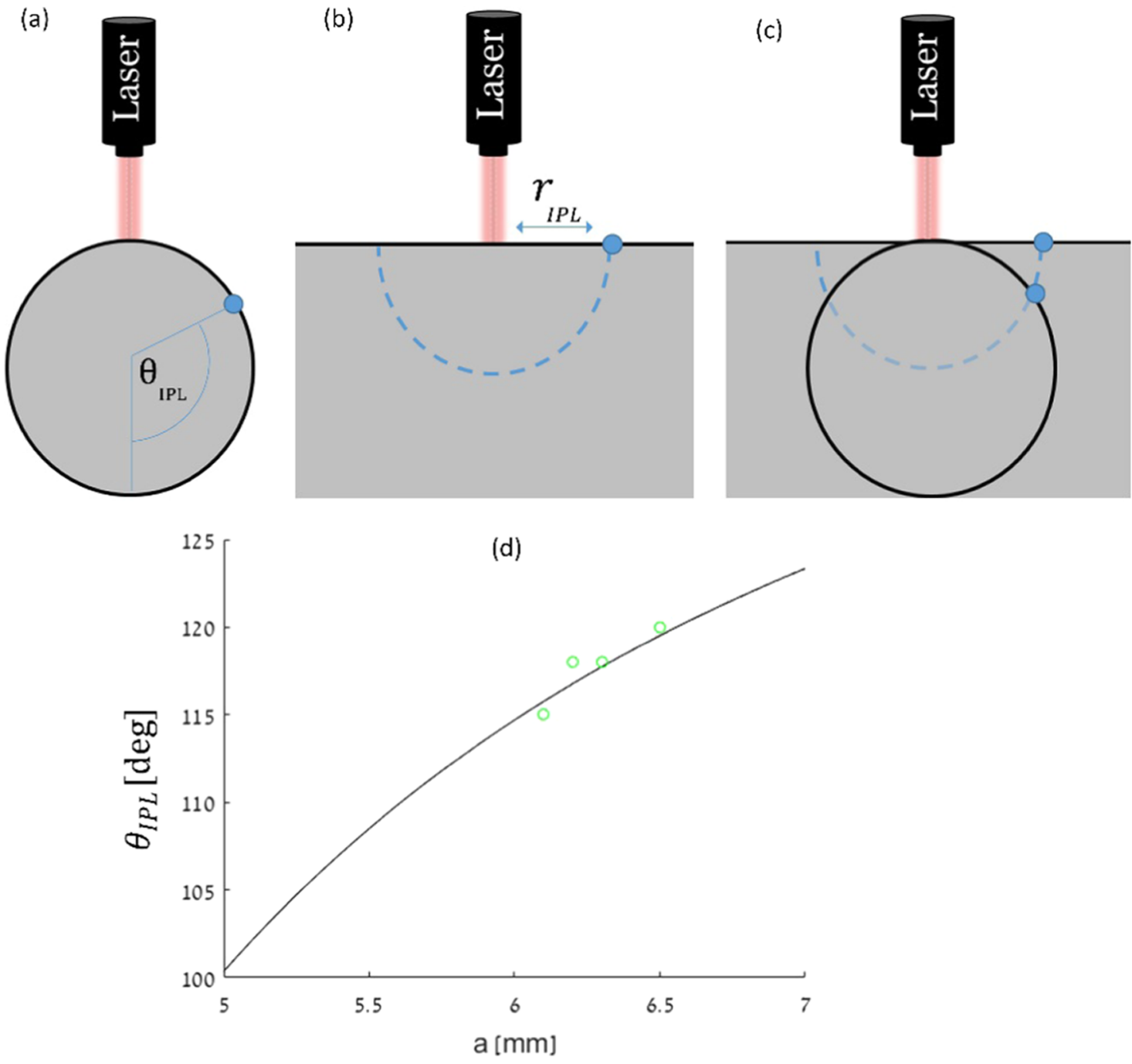

Figure 11. Schemas of (a) an irradiated cylindrical tissue cross section and the location of $\theta_{\mathrm{IPL}}$ (b) an irradiated semi-infinite tissue and the location of $r_{\mathrm{IPL}}$ (c) both geometries on the same coordinate system, (d) comparison between theoretical and experimental results; the relation between $\theta_{\mathrm{IPL}}$ and tissue radius $a$ is calculated by intersection with $r_{\text {IPL }}$ (curve) and experimental results of human fingers (dots).

to extract an accurate physiological parameter from the absorption regardless of the additional optical parameters' effect.

By using the FSP method, we show the effect of optical properties on the FSP and the IPL point that is not influenced by the reduced scattering coefficient in a cylindrical medium. We present in this article the existence of the IPL phenomenon in theory, simulations, and experimental results using tissuelike phantoms and finally in vivo measurements.

In oxygen tissue assessment, the transmitted light is described by the modified Beer-Lambert law, ${ }^{21} I=I_{0}$ $\mathrm{e}^{-\left(\mu_{\mathrm{a}}(\lambda) l(\lambda)+G\right)}$, where $l$ is the optical path length that depends on the absorption and scattering coefficients and $G$ is a geometric factor. When measuring at several wavelengths, $l$ changes. The existing methods suffer from an inherent error due to these differences in the optical path length, which must be calibrated externally via in vitro measurements. The calibration process adds an error that is too high for some clinical problems; ${ }^{23,24}$ therefore, it is challenging to extract the needed parameter (e.g., $\mathrm{SpO}_{2}$ ) correctly. ${ }^{25}$

The IPL point overcomes this problem because the different sources undergo the same optical path length even though they experience different reduced scattering coefficients.
Moreover, this phenomenon of a geometric location, where the intensity is independent of the reduced scattering coefficients, appears in a semi-infinite medium as well. We demonstrated that this phenomenon occurs through diffusion theory, MC simulation, and experiments using phantoms as well as in vivo measurements in the DR system. There are researchers who examined the light scattering on the basis of diffusion theory and an equivalent isotropic source and an extrapolated boundary, ${ }^{17}$ and their solution agrees with ours.

In addition, a similar geometric phenomenon was reported by others ${ }^{13}$ about a distance from the light source that is neutralized by the effect of the phase function. This optical parameter has an influence on calculations of the reflected intensity beside a specific location. This location can also be called an IPL point where reflectance is not dependent on the phase function.

The correct choice of a measurement point allows the neutralization of the scattering effect and as a result enables us to measure other optical parameters accurately. The use of this unique behavior can predict more accurate measurements in the biomedical field. The measurement of the light intensity at the IPL point, which is independent of the tissue scattering, can improve accuracy in two ways: as a self-calibration point for 
another measurement point on the FSP in a single wavelength or by measuring the absorption at the IPL point that neutralizes the scattering at two wavelengths.

\section{COMPUTATIONAL METHODS AND EXPERIMENTAL SECTION}

6.1. Monte Carlo Simulation. The most common model of simulating light-tissue interactions is the MC simulation. MC simulations are recommended for regions close to the source. We investigated the dependency of the IPL point in the case of different tissue diameters. As we explained in our previous work, ${ }^{26}$ we simulated a beam of photons that enters the circular cross section of tissue parallel to the $z$ direction. The propagation path of each photon is calculated from the scattering constant, assuming that the absorption is negligible. The values of the reduced scattering coefficients $\left(\mu_{\mathrm{s}}{ }^{\prime}\right)$ were in the range of the human skin values. ${ }^{6}$ In addition, we used a MC simulation from a semi-infinite medium that Prof. Jacques shared with us to deepen and enrich the research.

We simulated the FSP of a circular cross section of a tissue via $\mathrm{MC}$ method with relevant assumptions and changes. We chose several reduced scattering coefficients and different tissue diameters. This simulation is based on the assumption that all photons reaching the tissue begin as ballistic photons. At each step $(\mathrm{d} r)$ of the photon, the new direction $\left(\theta_{\text {new }}\right)$ was calculated using the current photon's direction $\left(\theta_{\text {old }}\right)$ and the probability a photon would scatter, $1-\exp \left(-\mu_{\mathrm{s}}^{\prime} \mathrm{d} r\right)$. In the case of a scattering event, its new direction was calculated using $\theta_{\text {new }}=$ $\theta_{\text {old }}+s \times \cos ^{-1} g$, where $s$ is a random number from the group $[-1,1]$. The location of a photon that arrived to the boundary of the cylindrical tissue was saved as the exit angle.

6.2. Experiments. 6.2.1. FSP Setup. The setup includes a continuous wave illumination using a $\mathrm{He}-\mathrm{Ne}$ gas laser (Thorlabs, Newton, SA) with a wavelength of $\lambda=632.8 \mathrm{~nm}$, a maximum power of $8 \mathrm{~mW}$, and a laser beam waist of $\omega_{0}=$ $0.63 \mathrm{~mm}$. In addition, for in vivo measurement, a pigtailed NIR laser (Oxxius S.A., France) was used with $\lambda=785 \mathrm{~nm}$. We use a portable fixed gain silicon detector as a PD. The PD has an active area of $0.8 \mathrm{~mm}^{2}$, and it is placed on a rotation stage to enable the full scattering profile measurements. The stage can be rotated around a tube with an accuracy of $1^{\circ}$. The voltage is measured every $5^{\circ}$, where $0^{\circ}$ is the full transmission and $180^{\circ}$ is the full reflection.

6.2.2. Phantoms. Cylindrical tissuelike phantoms were prepared on the basis of intra-lipid (IL) in various concentrations to measure the influence of the reduced scattering coefficient on the FSP experimentally. The IL concentration is calculated according to the reduced scattering coefficients for each phantom, ${ }^{13}$ in the range of the human skin values of $8-26 \mathrm{~cm}^{-1}$, similar to that in the MC simulation. The liquid phantoms were placed in test tubes with different diameters. They were measured from 0 to $145^{\circ}$, whereas the remaining angles were not measurable because of the blocking of the light source by the detector.

6.2.3. DR Measurements System. The diffusion reflectance (DR) method is a simple and safe technology for extracting optical parameters using the light intensity at different distances $\rho$ (light source-detector separation). The DR system (NEGOH-OP Technologies, Israel), as was previously described by us, ${ }^{27}$ contains two sources, 650 and $780 \mathrm{~nm}$ using an optic fiber with a diameter of $125 \mu \mathrm{m}$. A portable photodiode was deposited at different distances $(\rho)$ from the source on the sample surface in a micrometer scale. The photodiode had a cross-section diameter of $1 \mathrm{~mm}^{2}$, the reflected intensity was measured.

The in vivo measurements using the DR system were carried out on a C57BL male mouse (aged 4.5 months). The mouse was anesthetized by injecting intraperitoneally $200 \mu \mathrm{L}$ of a mixture of ketamine $(10 \mathrm{mg} / \mathrm{mL})$ and xylazine $(2 \mathrm{mg} / \mathrm{mL})$. The hair on the neck facing the laser was removed before the measurements.

This study received institutional approval from the Bar Ilan Institute for Nanotechnology and Advanced Materials Institutional Animal Care and Use Committee. All in vivo measurements were performed under appropriate anesthesia: the mice barrier-controlled facility followed the guidelines of Bar Ilan's Institute for Nanotechnology and Advanced Materials Animal Care and Use Committee. The mice were inspected daily by the veterinarian, who handled the appropriate tests and treatment protocols as required. All research protocols were followed strictly by the veterinarian. All major procedures were performed in the surgical facilities using general anesthesia and standard aseptic surgical techniques.

\section{ASSOCIATED CONTENT}

\section{Supporting Information}

The Supporting Information is available free of charge on the ACS Publications website at DOI: 10.1021/acsomega.8b00018.

Video file for the IPL points that are not influenced by the scattering properties of the medium in the two mentioned geometries that merge to the same distance from the light source (AVI)

$\mathrm{MC}$ simulation for a higher range of reduced scattering coefficients (Figure S1) (PDF)

\section{AUTHOR INFORMATION}

\section{Corresponding Author}

*E-mail: Dror.Fixler@biu.ac.il.

ORCID

Dror Fixler: 0000-0003-0963-7908

Notes

The authors declare no competing financial interest.

\section{REFERENCES}

(1) Yaroslavsky, A.; Schulze, P.; Yaroslavsky, I.; Schober, R.; Ulrich, F.; Schwarzmaier, H. Optical properties of selected native and coagulated human brain tissues in vitro in the visible and near infrared spectral range. Phys. Med. Biol. 2002, 47, 2059-2073.

(2) Schmitt, J. M. Optical coherence tomography (OCT): a review. IEEE J. Sel. Top. Quantum Electron. 1999, 5, 1205-1215.

(3) Handgraaf, H. J.; Verbeek, F. P.; Tummers, Q. R.; Boogerd, L. S.; van de Velde, C. J.; Vahrmeijer, A. L.; Gaarenstroom, K. N. Real-time near-infrared fluorescence guided surgery in gynecologic oncology: a review of the current state of the art. Gynecol. Oncol. 2014, 135, 606613.

(4) Talari, A. C. S.; Movasaghi, Z.; Rehman, S.; Rehman, I. U. Raman spectroscopy of biological tissues. Appl. Spectrosc. Rev. 2015, 50, 46111.

(5) Fixler, D.; Duadi, H.; Ankri, R.; Zalevsky, Z. Determination of coherence length in biological tissues. Lasers Surg. Med. 2011, 43, 339-343.

(6) Jacques, S. L. Optical properties of biological tissues: a review. Phys. Med. Biol. 2013, 58, R37-R61.

(7) Tromberg, B. J.; Shah, N.; Lanning, R.; Cerussi, A.; Espinoza, J.; Pham, T.; Svaasand, L.; Butler, J. Non-invasive in vivo characterization of breast tumors using photon migration spectroscopy. Neoplasia $2000,2,26-40$. 
(8) Feder, I.; Duadi, H.; Fixler, D. Experimental system for measuring the full scattering profile of circular phantoms. Biomed. Opt. Express 2015, 6, 2877-2886.

(9) Duadi, H.; Feder, I.; Fixler, D. Linear dependency of full scattering profile isobaric point on tissue diameter. J. Biomed. Opt. 2014, 19, No. 026007.

(10) Mourant, J. R.; Bigio, I. J.; Jack, D. A.; Johnson, T. M.; Miller, H. D. Measuring absorption coefficients in small volumes of highly scattering media: source-detector separations for which path lengths do not depend on scattering properties. Appl. Opt. 1997, 36, 56555661 .

(11) Kumar, G.; Schmitt, J. Optimal probe geometry for near-infrared spectroscopy of biological tissue. Appl. Opt. 1997, 36, 2286-2293.

(12) Yariv, I.; Haddad, M.; Duadi, H.; Motiei, M.; Fixler, D. New optical sensing technique of tissue viability and blood flow based on nanophotonic iterative multi-plane reflectance measurements. Int. J. Nanomed. 2016, 11, 5237-5244.

(13) Calabro, K. W.; Bigio, I. J. Influence of the phase function in generalized diffuse reflectance models: review of current formalisms and novel observations. J. Biomed. Opt. 2014, 19, No. 075005.

(14) Savo, R.; Pierrat, R.; Najar, U.; Carminati, R.; Rotter, S.; Gigan, $\mathrm{S}$. Observation of mean path length invariance in light-scattering media. Science 2017, 358, 765-768.

(15) Jacques, S. L.; Pogue, B. W. Tutorial on diffuse light transport. J. Biomed. Opt. 2008, 13, No. 041302.

(16) Ankri, R.; Fixler, D. Gold nanorods based diffusion reflection measurements: current status and perspectives for clinical applications. Nanophotonics 2017, 6, No. 33.

(17) Zhang, A.; Piao, D.; Bunting, C. F.; Pogue, B. W. Photon diffusion in a homogeneous medium bounded externally or internally by an infinitely long circular cylindrical applicator. I. Steady-state theory. J. Opt. Soc. Am. A 2010, 27, 648-662.

(18) Fixler, D.; Nayhoz, T.; Ray, K. Diffusion reflection and fluorescence lifetime imaging microscopy study of fluorophoreconjugated gold nanoparticles or nanorods in solid phantoms. ACS Photonics 2014, 1, 900-905.

(19) Ankri, R.; Ashkenazy, A.; Milstein, Y.; Brami, Y.; Olshinka, A.; Goldenberg-Cohen, N.; Popovtzer, A.; Fixler, D.; Hirshberg, A. Gold Nanorods based air scanning electron microscopy and diffusion reflection imaging for mapping tumor margins in squamous cell carcinoma. ACS Nano 2016, 10, 2349-2356.

(20) Sabino, C. P.; Deana, A. M.; Yoshimura, T. M.; da Silva, D. F.; França, C. M.; Hamblin, M. R.; Ribeiro, M. S. The optical properties of mouse skin in the visible and near infrared spectral regions. J. Photochem. Photobiol., B 2016, 160, 72-78.

(21) Delpy, D. T.; Cope, M.; van der Zee, P.; Arridge, S.; Wray, S.; Wyatt, J. Estimation of optical pathlength through tissue from direct time of flight measurement. Phys. Med. Biol. 1988, 33, 1433.

(22) Duadi, H.; Feder, I.; Fixler, D. Near-infrared human finger measurements based on self-calibration point: simulation and in vivo experiments. J. Biophotonics 2017, No. e201700208.

(23) Nitzan, M.; Noach, S.; Tobal, E.; Adar, Y.; Miller, Y.; Shalom, E.; Engelberg, S. Calibration-free pulse oximetry based on two wavelengths in the infrared - a preliminary study. Sensors 2014, 14, 74207434.

(24) Pickering, J. W.; Prahl, S. A.; Van Wieringen, N.; Beek, J. F.; Sterenborg, H. J.; Van Gemert, M. J. Double-integrating-sphere system for measuring the optical properties of tissue. Appl. Opt. 1993, 32, 399-410.

(25) Duadi, H.; Nitzan, M.; Fixler, D. Simulation of oxygen saturation measurement in a single blood vein. Opt. Lett. 2016, 41, $4312-4315$.

(26) Duadi, H.; Fixler, D.; Popovtzer, R. Dependence of light scattering profile in tissue on blood vessel diameter and distribution: a computer simulation study. J. Biomed. Opt. 2013, 18, 111408.

(27) Ankri, R.; Taitelbaum, H.; Fixler, D. Reflected light intensity profile of two-layer tissues: phantom experiments. J. Biomed. Opt. 2011, 16, No. 085001. 